thrombocytosis and other prognostic factors DFS and OS was the aim of study.

Methods: Aim of this study was to analyze the impact of pretreatment thrombocytosis to the prognosis of EC cases. Medical records of EC cases who underwent complete surgical staging, treatment and follow up in Bhumibol Adulyadej Hospital (BAH) during January 2008 and December 2020 were reviewed. Demographic data included age, body weight, height, body mass index, histology and grading, cancer stage, MI, LN metastasis, LVSI, peritoneal washing cytology, platelet count, recurrence, death and follow up time were collected. Associations of thrombocytosis and prognostic factors were analyzed with DFS and OS. A total of 222 cases were included in study. Mean age of participant in the present study was 56.16 years old. One third (88/222) of cases had age more than 60 years old. Ninety percent of histopathology was reported as endometrioid subtype. MI more than half of thickness, well and moderate differentiation (G1, 2) and advanced cancer stage (FIGO stage III, IV) were reported at $44.1 \%, 79.4 \%$, and $22.5 \%$, respectively. Thrombocytosis was reported at $8.1 \%$. Aggressive histopathology, advanced cancer stage (FIGO stage III, IV), MI more than half of thickness, LN metastasis, present of LVSI and thrombocytosis was significantly correlated with DFS. Advanced cancer stage (FIGO stage III, IV) and thrombocytosis had significant adverse effect on OS. In conclusion, advanced endometrial cancer stage and thrombocytosis were significant adverse prognostic factor.

Poster (E19)

Endometrial Hyperplasia, Endometrial Intra-epithelial Neoplasia, and Endometrial Cancer

https://doi.org/10.3802/jgo.2021.32.S1.E19

\section{The utility of preoperative serum CA125 combined with HE4 to predict lymph node metastasis in endometrial cancer: a cross- sectional study}

\footnotetext{
Sansanee Angsathapon, Nisa Pruksaritanond

Rajavithi Hospital, Bangkok, Thailand (nisa3054@gmail.com)
}

Objective: To investigate the diagnostic performance of the serum cancer antigen 125 (CA125), human epididymis protein 4 (HE4), a combination of CA125 and HE4, and a risk of ovarian malignancy algorithm (ROMA) in the pre-operative prediction of high risk of lymph node metastasis in patients with earlystage endometrial cancer (EC).

Methods: A cross-sectional study of data for patients with early-stage endometrioid EC treated surgically at Rajavithi
Hospital between April 2020 and April 2021 was commenced. The preoperative serum levels of CA125 and HE4 were measured and analyzed by clinicopathological characteristics. The receiver operating characteristic (ROC) curves were generated to determine the optimal cutoff values of CA125, HE4, and ROMA levels with optimum sensitivity and specificity for predicting lymph node metastasis.

Results: Eighty-six patients with surgically staged EC were identified. Lymph node involvement was detected in 9 patients $(10.5 \%)$ with a systemic lymph node dissection. The median serum CA125, HE4, and ROMA levels were significantly higher in EC patients with the presence of lymph node metastasis than those who did not $(\mathrm{p}<0.05)$. Based on the ROC curve, both serum markers showed good discrimination for the prediction of lymph node metastasis, with an optimal cutoff value of $35 \mathrm{U} /$ $\mathrm{mL}$ for CA125 (area under curve [AUC] $=0.789 ; 95 \%$ confidence interval $[\mathrm{CI}]=0.647-0.932), 200 \mathrm{pMol} / \mathrm{L}$ for HE4 (AUC=0.825; $95 \% \mathrm{CI}=0.700-0.950$ ), and $60 \%$ for ROMA (AUC $=0.856 ; 95 \%$ $\mathrm{CI}=0.720-0.982)$. At the optimal cutoff value, HE4 showed the highest sensitivity $(88.9 \%)$ whereas the combination of CA125 and HE4 had the highest specificity $(87.0 \%)$.

Conclusion: Pre-operative combining CA125 and HE4 level is associated with an increased risk of having lymph node metastasis in patients with early-stage EC. This biomarker panel can be used as a predictive marker and guidance for identifying EC patients who might most benefit from full staging surgery with lymphadenectomy.

Poster (E20)

Endometrial Hyperplasia, Endometrial Intra-epithelial Neoplasia, and Endometrial Cancer https://doi.org/10.3802/jgo.2021.32.S1.E20

\section{A personalised predictive 3-year overall survival for patients with uterine carcinosarcoma at tertiary care hospital of southern Thailand}

\section{Ingporn Jiamset," Pacharadol Taweerat}

Prince of Songkla University, Songkhla, Thailand (ing_ing01@hotmail.com)

Objective: To develop a 3-year overall survival (OS) nomogram to predict outcome of surgically staged patients with uterine carcinosarcomas (UCSs).

Methods: This retrospective study, the clinicopathological, treatment data, and oncological outcomes of 69 UCSs patients between January 2002 and September 2018 were analyzed. We identified and integrated significant prognostic factors for OS to develop a nomogram. The concordance probability (CP) was 
used as a precision measure. The model was internally validated with bootstrap samples to correct overfitting.

Results: The median follow-up times was 19.4 months (range, 0.77-106.13 months), and the 3-year OS was 41.8\% ( $95 \%$ confidence interval $[\mathrm{CI}]=29.9 \%-58.3 \%$ ). We found International Federation of Gynecology and Obstetrics (FIGO) stage, and adjuvant chemotherapy were independent factors for OS. The $\mathrm{CP}$ of the nomogram integrating with body mass index (BMI), FIGO stage and adjuvant chemotherapy was 0.72 $(95 \% \mathrm{CI}=0.70-0.75)$. The calibration curves for probability of 3-year OS also demonstrated agreement between nomogram prediction and observation data.

Conclusion: The nomogram to predict 3-year OS of UCSs after surgery with simple parameters include BMI, FIGO stage, and adjuvant chemotherapy was implemented in a nomogram and provides accurate prediction of individual patients' prognosis useful for patient counselling and deciding on follow-up strategies.

Poster (E21)

Endometrial Hyperplasia, Endometrial Intra-epithelial Neoplasia, and Endometrial Cancer

https://doi.org/10.3802/jgo.2021.32.S1.E21

\section{A validation study of a modified TCGA classification for patients with endometrial cancer treated with radical surgery and adjuvant chemotherapy}

\footnotetext{
Hiroyuki Yamazaki, ${ }^{1,}$ Kanako Hatanaka, ${ }^{2}$ Hiroshi Asano, Matsuno Yoshihiro, ${ }^{3}$ Yutaka Hatanaka, ${ }^{2}$ Hidemichi Watari

'Department of Obstetrics and Gynecology, Graduate School of Medicine and Faculty of Medicine, Hokkaido University, Sapporo, Japan (cvm5396@elms.hokudai.ac.jp)

${ }^{2}$ Center for Development of Advanced Diagnostics, Research Division of Genome Companion Diagnostics, Hokkaido University Hospital, Sapporo, Japan

${ }^{3}$ Department of Surgical Pathology, Hokkaido University Hospital, Sapporo, Japan
}

Objective: The Cancer Genome Atlas (TCGA) molecular classification for endometrial cancer is expected to propose the most appropriate treatment strategy for each patient. However, the prognostic stratifications remain unclear when adjuvant chemotherapy is applied. This study aimed to validate a modified TCGA classification, ProMisE, for patients who underwent adjuvant chemotherapy at intermediate or high risk of recurrence.

Methods: From 2003 to 2015, the patients who underwent systematic lymphadenectomy were enrolled. The patients were classified by ProMisE using immunohistochemistry for mismatch repair-related molecules (MLH1, MSH2, MSH6, PMS2) and p53, and direct sequencing for hotspot mutations in POLE (exon 9, 13, and 14). The 5-year disease-specific survival rates (5y-DSS) were estimated by the Kaplan-Meier method. Results: A total of 182 patients were analyzed. The median age and follow-up period were 57.4-year-old and 105 months. The patients categorized to stage I, II, III, and IV were 97, 23, 51, and 11, respectively. One hundred twenty-two patients were diagnosed as endometrioid carcinoma (grade 1-2), and 60 were high-grade types, including endometrioid (grade 3), serous, and clear cell carcinomas. Based on ProMisE, 57 patients were categorized into mismatch-repair deficiency (MMR-d), 45 into POLE-EDM (exonuclease domain mutations), 15 into p53 wildtype, and 4 into p53 abnormal. The 5y-DSS was $92.9 \%, 100 \%$, $91.1 \%$, and $75.0 \%$, respectively.

Conclusion: We conclude that ProMisE can stratify the prognosis even when adjuvant chemotherapy was applied for intermediate- and high-risk for recurrence. The 5y-DSS of MMR-d patients seemed better than previous reports, partly because adjuvant chemotherapy could improve their prognosis.

Poster (E22)

Endometrial Hyperplasia, Endometrial Intra-epithelial Neoplasia, and Endometrial Cancer https://doi.org/10.3802/jgo.2021.32.S1.E22

\section{Association between high body mass index and endometrial pathology in premenopausal women with abnormal uterine bleeding}

\author{
Ruangsak Lertkhachonsuk," Nuttida Mahakit, \\ Woranit Apikulprapa, Patou Tantbairojn \\ Chulalongkorn University, Bangkok, Thailand (ruangsak@chula.md)
}

Objective: To find out the correlation between body mass index and endometrial pathology in premenopausal women with abnormal uterine bleeding.

Methods: A cross-sectional study was conducted in King Chulalongkorn Memorial Hospital during 1 January to 31 December 2019. All cases of abnormal uterine bleeding in premenopausal women who had endometrial pathology were recruited. All endometrial histopathology had been reviewed. Patient clinical profiles were recorded. Body mass index (BMI) was calculated by using weight in kilogram divided by square of height in meters. Then all cases were categorized by Asia Pacific BMI classification into underweight $\left(<18.5 \mathrm{~kg} / \mathrm{m}^{2}\right)$, normal weight (18.5-22.9 kg/m²), overweight (23-24.9 kg/m²) and obese $\left(>25 \mathrm{~kg} / \mathrm{m}^{2}\right)$. The pathological reports were classified into 3 groups: normal epithelium, endometrial hyperplasia, and endometrium cancer. Statistical analysis was done SPSS version 